\title{
Accurate laser scanner to camera calibration with application to range sensor evaluation
}

\author{
Peter Fuersattel ${ }^{1,2^{*}}$ (D), Claus Plank ${ }^{3}$, Andreas Maier $^{1}$ and Christian Riess $^{1}$
}

\begin{abstract}
Multi-modal sensory data plays an important role in many computer vision and robotics tasks. One popular multi-modal pair is cameras and laser scanners. To overlay and jointly use the data from both modalities, it is necessary to calibrate the sensors, i.e., to obtain the spatial relation between the sensors.

Computing such a calibration is challenging as both sensors provide quite different data: cameras yield color or brightness information, laser scanners yield 3-D points. However, several laser scanners additionally provide reflectances, which turn out to make calibration to a camera well feasible. To this end, we first estimate a rough alignment of the coordinate systems of both modalities. Then, we use the laser scanner reflectances to compute a virtual image of the scene. Stereo calibration on the virtual image and the camera image are then used to compute a refined, high-accuracy calibration.

It is encouraging that the accuracies in our experiments are comparable to camera-camera stereo setups and outperform another of other target-based calibration approach. This shows that the proposed algorithm reliably integrates the point cloud with the intensity image. As an example application, we use the calibration results to obtain ground-truth distance images for range cameras. Furthermore, we utilize this data to investigate the accuracy of the Microsoft Kinect V2 time-of-flight and the Intel RealSense R200 structured light camera.
\end{abstract}

Keywords: Laser scanner, Range camera, RealSense R200, Kinect V2

\section{Introduction}

Finding the spatial relation between a laser scanner and a 2-D or 2.5-D camera is crucial for sensor data fusion. Knowing this relation enables a multitude of applications, for example coloring the point cloud, the generation of textured meshes, or the creation of high accuracy ground truth for range cameras. The method proposed in this work has been specifically designed for generating reference distances for range camera evaluation. Nonetheless, the approach is not limited to this application and can also be used to calibrate a common 2-D camera to a laser scanner.

Range cameras find widespread use, for example in the field of robotics [1], in space [2, 3], automation in

\footnotetext{
*Correspondence: peter.fuersattel@fau.de

${ }^{1}$ Pattern Recognition Lab, University of Erlangen - Nuremberg, Martensstrasse 3, Erlangen, Germany

${ }^{2}$ Metrilus GmbH, Henkestrasse 91, Erlangen, Germany

Full list of author information is available at the end of the article
}

logistics [4] or in augmented reality devices like the Google Tango phones. The major problem with these sensors is their limited accuracy. This gives rise to thorough camera evaluations with respect to accuracy and other individual camera characteristics that influence the range measurements.

Several studies that investigate the accuracies and error characteristics of range cameras have been presented in the past. Rauscher et al. [5] analyze range cameras with respect to their applicability to robotics. Yang et al. presented a detailed study on the Kinect V2 [6]. Fuersattel et al. evaluated multiple time-of-flight cameras with respect to different error sources [7]. Wasenmüller and Stricker compare the structured light Kinect V1 camera to the time-of-flight-based Kinect V2 camera [8].

Quantitative evaluation of range cameras requires scenes with ground truth distance measurements. Nair et al. state three methods to acquire such ground truth [9]: 
- Computed from a calibration pattern and known camera intrinsic parameters

- Computed from a calibration pattern as seen from a second high-resolution camera with known intrinsic parameters and known spatial relation to the evaluated camera.

- Measured with an additional, highly accurate 3-D sensor (e.g., a laser scanner) with known spatial relation to the evaluated camera.

The first two approaches have limited information value as they typically provide reference distances only for planar regions. Moreover, the accuracy of the ground truth quickly degrades as the distance between camera and calibration pattern increases.

A laser scanner mitigates both issues. Laser scanners typically provide high-accuracy point clouds of a scene for larger operating ranges than camera-based solutions. Also, this distance information can be obtained for arbitrary, not necessarily planar, surfaces. However, to leverage laser scanner point clouds for range camera evaluation, it is necessary to calibrate the laser scanner to the camera.

In this paper we propose a method for solving this task. Starting from a scene that shows multiple calibration patterns, e.g., checkerboards, we show how stereo calibration methods can be used to obtain the rotation and translation between the sensors. We aim at calculating the spatial relation based on a single point cloud/camera image pair, as acquiring densely sampled point clouds can take up to multiple minutes.

First, a virtual image of the point cloud has to be generated. It is important that this image shows all calibration patterns without occlusions. Thus, we demonstrate how the laser scanner's unordered point cloud can be transformed such that it is approximately aligned with the coordinate system of the camera. From this transformed point cloud, a virtual image is generated. In this image, the pixel intensities are derived from the reflectivity data that is associated with the individual 3-D point measurements.

The reflectivity data quantifies the amount of light that is reflected from a point in the scene back to the laser scanner. Therefore, the strong contrast of the calibration patterns also results in strong variations of the reflectivity data. By detecting the calibration patterns in both the virtual and the camera image, point correspondences for the two sensors can be obtained with sub-pixel accuracy. Finally, these corresponding points are used as input to established stereo calibration algorithms to obtain the spatial relation between both sensors. Note that it is necessary that the scene is sampled densely, such that at least one 3-D point measurement can be mapped to each pixel of the virtual image. Dense point clouds are required for example calculating accurate meshes of the scene, or like in our application, for generating ground-truth distance measurements for range cameras. In this work, we exploit the high sampling density to achieve even more accurate calibration results than current state-of-the-art methods.

The proposed method is evaluated with multiple data sets from four different range cameras. We show both qualitatively and quantitatively that the presented method aligns the coordinate systems accurately and, furthermore, considerably outperforms the baseline method. In image domain, misalignments of less than 0.2 pixels are achieved. For corresponding 3-D coordinates in the scene, the mean error is as small as $1.3 \mathrm{~mm}$.

The contributions of this work consist of two parts.

1. We present an automatic method for calibrating a laser scanner to a camera. This method enables the user to estimate the spatial relation between the two sensors with a single shot of a scene, which contains only a small number of calibration patterns. The applicability of the proposed method is shown for four different camera-laser scanner setups.

2. We use the calibration technique to present accuracy evaluations for different range camera technologies: the Microsoft Kinect V2 time of flight camera and Intel RealSense R200 structured light cameras.

In Section 2 we present related work. Detailed information on the proposed algorithm can be found in Section 3. The evaluation of the performance of the presented approach and the range camera evaluation results are presented in Section 4. Section 5 summarizes and concludes this work.

\section{Related work}

Several approaches exist for calibration of laser scanners to cameras. Oftentimes, these methods are categorized by the type of laser scanner they operate on, namely methods for line scanners and methods for 2.5-D laser scanners.

For calibration of a 2-D laser line scanner to a camera, Zhang et al. [10] proposed a method that makes use of checkerboards for aligning the coordinate systems of both modalities. The method requires multiple acquisitions from different positions to establish sufficiently many constraints for nonlinear optimization. More recently, Kassir et al. [11] propose an automatic toolbox that builds on top of the well-known Camera Calibration Toolbox for Matlab. The toolbox is extended by detection algorithms for both checkerboards in the camera images and lines in the laser scanner data. In an iterative process, the spatial relation is optimized such that the detected lines match the planes of the calibration pattern. Zhou [12] presented a numerically more stable approach that also uses 
plane-line correspondences to constrain the estimation. This method also requires fewer plane-line pairs than the method by Zhang et al. [10].

Line laser scanners obtain range information only for a single scanline. In the context of range camera evaluation, this information is not sufficient. Instead, dense 2.5-D point clouds are preferable. For example, Unnikirshnan et al. [13] published an interactive Matlab toolbox to calculate the spatial relation between a camera and a 2.5-D dense point cloud. The authors recommend using at least 15 to 20 images. In each of the images, the calibration pattern region has to be delimited manually by drawing a polygon that encloses the area. In contrast, we find the planar segments of the calibration pattern automatically. With multiple patterns in the scene, an accurate calibration from a single shot is possible. The method proposed by Geiger et al. [14] obtains the spatial relation based on a single shot of a scene that shows multiple checkerboard patterns. Based on the checkerboards and planar segments in the scene, an initialization for a subsequent iterative closest point-based refinement is calculated. In contrast to their work, we incorporate the laser scanner amplitudes to reduce the impact of inaccuracies of the plane detection.

Ha et al. [15] propose a new, specifically constructed calibration pattern with a triangular hole to reduce the number of calibration images. While other methods typically require at least three different poses of calibration patterns, this method requires only two. Hoang et al. [16] also use a calibration pattern with a triangular hole. Their pattern is used to obtain 3-D/2-D correspondences for solving the perspective- $n$-point problem. Gong et al. [17] propose to use as a calibration target three planes that form a trihedral. Based on at least two shots on such scenes, the relative transformation between the two sensor coordinate systems is estimated via nonlinear optimization. Although the method does not require special calibration targets, it still requires the user to define the planar region in the camera image. Our method requires three patterns, but these are off-the-shelf patterns without particular manufacturing requirements.

Moghadam et al. [18] estimate the spatial relation from line segments that can be detected both in the point cloud and in the camera image. Taylor and Nieto [19] find the spatial relation by maximizing the mutual information between the camera image and a virtual image, which is colored according to the direction of the point cloud's normals. The work presented in [20] extends this method by a more robust normal estimation algorithm. However, both methods share the same drawbacks: the usage of particle swarm optimization requires (a) the initial knowledge of the range of the extrinsic parameters and (b) a computationally expensive rendering of a virtual image for each particle in each iteration. In contrast, we propose to use scene reflectivity to generate virtual views, which enables the use of highly accurate calibration targets. Pandey et al. [21] also do not require a calibration target. The authors propose a calibration via minimizing the mutual information between the camera pixels and the laser scanner reflectivity information. This approach requires multiple views in order to obtain a smooth cost function that can be optimized robustly. Levinson and Thrun proposed a framework that monitors the accuracy of a calibrated camera-laser scanner setup while being in use [22]. If a miss-calibration is observed, the extrinsic parameters are corrected by finding the transformation which maximizes the overlap between edges in the image and in the point cloud. This approach requires multiple frames and varied scene geometry such that sufficient corresponding edges can be found and a smooth cost function can be obtained. Scott et al. [23] presented an approach for the calibration of a laser scanner and cameras setup that also exploits reflectivity information. The method is suited for setups that move through an environment, e.g., in an autonomous driving scenario. The authors relax the constraint that field of views at a single point in time must overlap. Instead, the authors assume that some overlap will occur at some later point in time due to motion of the rigid sensor setup. The abovementioned methods are particularly useful if no calibration pattern is present, e.g., outside a lab environment. However, the disadvantage of these approaches is the reduced accuracy compared to controlled lab setups.

\section{Laser scanner to camera calibration}

The basic concept of the proposed method is that we use stereo calibration on the camera intensities and the laser scanner reflectivities. Thus, the large reflectivity differences of checkerboards allow to compute rotation and translation between the coordinate systems of both sensors. Classic stereo calibration expects two input images. The first input image is the intensity image from the range camera. The second input image, from the laser scanner, needs to be computed: the laser scanner measures an unordered 2.5-D point cloud that has to be rendered to a virtual image. In order to perform the rendering, a viewing direction onto the point cloud has to be defined. Good viewing directions avoid occlusions, and yield a balanced, unencumbered picture of the checkerboards in the scene. Figure 1 shows a negative example, i.e., a virtual image computed from a bad viewing direction. Here, only two of the checkerboard patterns can be detected completely. The corners of the left checkerboard cannot be found automatically due to the pose of the pattern. Additionally, this pattern occludes some parts of the big pattern in its background. Such occlusions can make calibration 


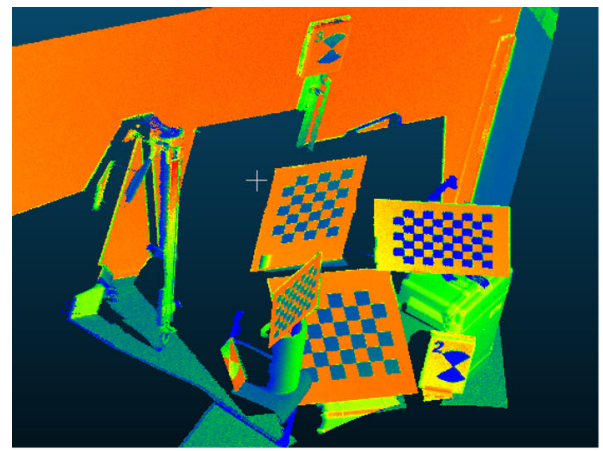

a

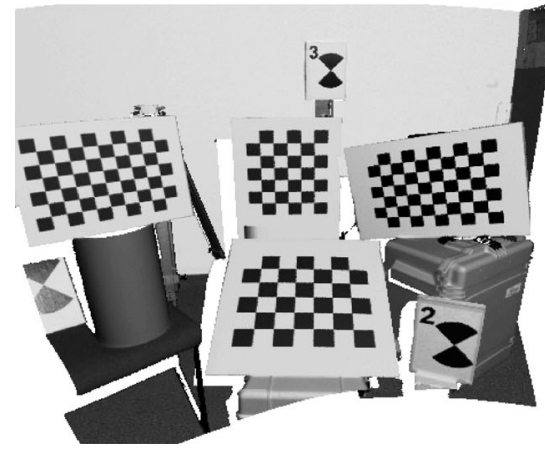

C Virtual reflectance image

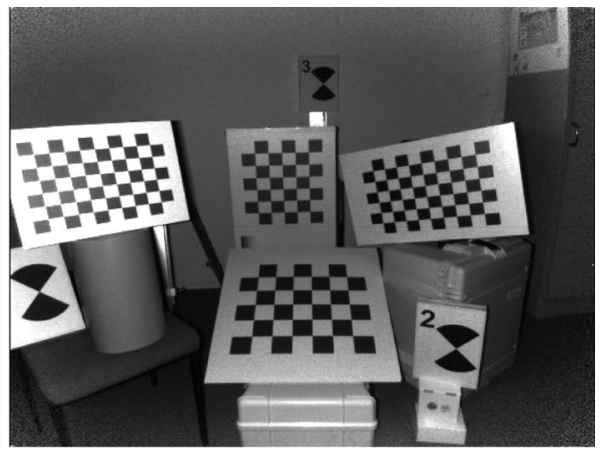

b Kinect V2 amplitude image

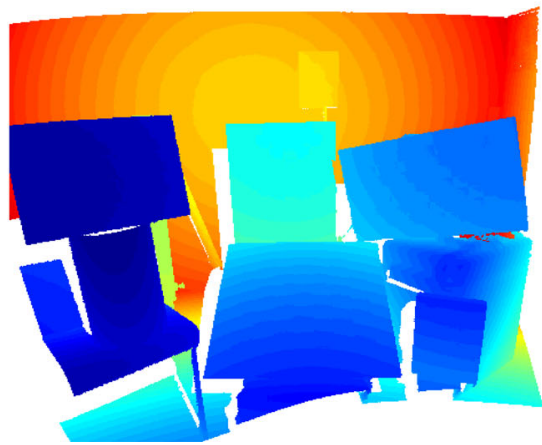

d Virtual distance image

Fig. 1 Examples from a Kinect V2 / laser scanner setup. a Laser scanner data, visualized in a point cloud viewer. b Amplitude image of the Kinect V2 for reference. $\mathbf{c}, \mathbf{d}$ Virtual reflectance and distance images of the point cloud as seen from the camera position

much more difficult or even cause complete failure. We mitigate this problem by computing a virtual image that shows the calibration patterns from an angle similar to the observing camera. It is likely that there is considerable overlap between such computed virtual image and the camera image. Thus, we seek a transformation that approximately aligns the camera and laser scanner coordinate systems.

In the next subsections, we present the full calibration algorithm in three steps, i.e.,

1. Finding the plane segments of the calibration patterns in the unordered point cloud

2. Calculation of an initial alignment for the two coordinate systems

3. Virtual image generation and estimation of a spatial relation via stereo calibration

\subsection{Finding the calibration patterns in the point cloud}

We assume that planes within certain size boundaries stem from calibration patterns. We first search such planes in the unordered point cloud obtained from the laser scanner. The algorithm uses the idea that a plane is characterized by a set of co-located points with surface normals pointing towards the same direction. We define neighborhoods for individual points and normal-based region growing.

In organized point clouds, the term neighborhood is often defined as the 4-connected or 8-connected neighborhood of a $(x, y)$ coordinate of a 2-D array. This association is not available with unorganized point clouds. In this work, we define the neighborhood of a point $\boldsymbol{p}_{i}$ as its $N_{o}$ closest points in a $L_{2}$ sense. These neighbors can be efficiently looked up by organizing the point cloud in a suitable data structure, for example an Octree. The normal $\boldsymbol{n}_{i}$ for a point $\boldsymbol{p}_{i}$ can be approximated by fitting a plane to the point and its $N_{o}$ neighbors. This can be done efficiently by calculating the eigenvalue decomposition of the covariance matrix of these points [24].

Laser scanner data exhibits a relatively low noise level, and computing $\boldsymbol{n}_{i}$ from multiple points further reduces noise in the estimated normals. Yet, inspection of the normals still exhibits some unwanted variations. Therefore, we apply a modified version of a bilateral smoothing filter for organized point clouds [25]. The filter can be rewritten such that it operates on neighborhoods in unorganized point clouds. The smoothed 3-D point $\boldsymbol{p}_{i}^{\prime}$ is given by 


$$
\begin{array}{r}
\boldsymbol{p}_{i}^{\prime}=\left(\boldsymbol{p}_{i}+\sum_{j=0}^{N_{o}} w_{i j} \boldsymbol{p}_{j}\right) /\left(1+\sum_{j=0}^{N_{o}} w_{i j}\right), \\
\text { where } w_{i j}=\mathrm{e}^{\left(\alpha\left\|\boldsymbol{p}_{i}-\boldsymbol{p}_{j}\right\|_{2}\right)} \mathrm{e}^{\left(\beta\left\|\boldsymbol{n}_{i}-\boldsymbol{n}_{j}\right\|_{1}\right)},
\end{array}
$$

and $\boldsymbol{p}_{j}$ denotes the $j$ th neighbor of $\boldsymbol{p}_{i}$. The same smoothing can also be applied to each normal $\boldsymbol{n}_{i}$. The influence of the distance between points and the difference between normals is controlled with the parameters $\alpha$ and $\beta$.

The computed normals are used to segment planar segments in the point cloud. The algorithm begins with a random seed point to define a new planar segment. The seed point consists of a point coordinate and its associated normal. We perform breadth-first region growing on points with similar normals. In other words, all points in the neighborhood of the segment that have a similar normal are iteratively added to the current segment. Every time a new point has been added to a segment, its normal is updated to be the average normal of all supporting points. The similarity of two normals is determined by thresholding on their angular difference. In our experiments, a conservative threshold of $10^{\circ}$ has proven to work well. Segmentation stops if all points have been assigned to a segment label. The segmentation is similar to a previous method for approximate plane segmentation for organized point clouds [25].

With all points assigned to a planar segment, we select those segments that may represent calibration patterns. Assuming that the dimensions of the calibration patterns are known, it suffices to threshold on the sizes of the minimum oriented bounding boxes of all segments.

\subsection{Estimation of the initial spatial relation}

The initial transformation approximately aligns the coordinate system of the laser scanner and the camera. The initialization is calculated from the candidate planes and the planes derived from the checkerboard patterns visible in the camera image.

First, the checkerboard patterns have to be detected as accurately as possible in the camera image. In this work we use the detector proposed in [26]. With the known dimensions of the patterns and the intrinsic parameters of the camera, the 3-D coordinates of the calibration features (e.g., checkerboard corners) can be calculated. Correspondences between planes in the laser scanner and detected patterns in the camera image can be directly established if the calibration patterns can be uniquely identified by their size, and if the number of plane candidates matches the number of calibration patterns. Otherwise, these correspondences have to be estimated. The naive solution is to evaluate all possible permutations $K$ for planes and patterns and to choose the permutation that minimizes some error metric. If $N_{c}$ calibration patterns are used, then
$N_{c}$ plane candidates are drawn from all found plane candidates. The centroids of the plane segments $\boldsymbol{m}^{(p)}$ and the calibration patterns $\boldsymbol{m}^{(c)}$ are used as candidate correspondences to estimate a transformation $\mathcal{R}$. The optimum transformation $\mathcal{R}^{*}$ is the one that minimizes the following error metric

$$
\mathcal{R}_{i}^{*}=\underset{K}{\operatorname{argmin}} \sum_{i}^{N_{c}}\left\|\mathcal{R}\left(\boldsymbol{m}_{i}^{(c)}\right)-\boldsymbol{m}_{i}^{(p)}\right\| .
$$

The quality of a permutation is measured as the sum of the distances between the transformed centroids $\mathcal{R}\left(\boldsymbol{m}_{i}^{(c)}\right)$ and the centroids of the respective planar segments $\boldsymbol{m}_{i}^{(p)}$. Under the assumption that calibration patterns and planes have been accurately detected, then the minimum of the error metric corresponds to the best initialization. Note that other metrics could be employed here as well, e.g., metrics based on normal directions or combinations of normal directions and centroids. However, we found the metric in Eq. (3) to be sufficient, since the initialization requires only a rough estimate of the spatial relation. In our experiments, the number of permutations $K$ to search through was always low, since most plane candidates are already filtered out using the sizes of the calibration patterns.

The calculation above imposes mild constraints on the positions and orientations of the calibration patterns. These constraints are identical to the requirements for a robust stereo calibration and typically not difficult to satisfy: to obtain the most accurate results, the checkerboard poses must constrain all six degrees of freedom of the rigid body transformation. In practice, this means that the checkerboards need to point into different directions (see, e.g., Fig. 1b) and should cover as much area of the field of view as possible.

\subsection{Virtual view generation and refinement transformation via stereo calibration}

The initial, approximate alignment of the point cloud with the camera image can be used to perform stereo calibration. To this end, a virtual image is computed from the point cloud. Virtual image and camera image together are then used to obtain a second transformation that refines the initial relation between the sensors.

Brightness differences in the virtual image are created from reflectivity information at each point from the laser scanner. Strong reflectivity variations within the calibration pattern result in strong contrasts in the virtual image. This is particularly useful at the transition between black and white quads of the checkerboard pattern for calibration.

For generating the virtual view, a set of intrinsic parameters for the virtual camera is required. These parameters 
have to be defined such that the calibration patterns can be detected reliably. In this work, $\Pi(\boldsymbol{p})$ will be used to denote the projection from a point $\boldsymbol{p}$ in 3-D space onto a point $(u, v)$ image plane, with $\Pi$ containing the pinhole camera parameters as well as potential lens distortion parameters.

If the camera itself has a reasonable resolution, then their intrinsic parameters can also be used for the virtual camera. Otherwise, these parameters need to be selected manually. It is possible to distinguish two cases: first, if the camera resolution is very low, for example with time-offlight cameras, then a higher image resolution should be chosen. In contrast, if the image resolution of the camera is very high (e.g., > $1280 \times 1024)$, the point cloud might not be dense enough to provide good amplitude values for each pixel. In the latter case, the resolution needs to be adjusted to a smaller value.

The simplest method to obtain the intensity information for each pixel is to project all points of the point cloud onto the image plane. First, the intensities $C(\boldsymbol{p})$ of all points which are projected onto a single pixel coordinate $(u, v)$ have to be obtained. To this end, we define a function $\gamma(u, v, \boldsymbol{p})$, which indicates whether a point $\boldsymbol{p}$ is projected onto a particular $(u, v)$ coordinate or not.

$$
\gamma(u, v, \boldsymbol{p})=\left\{\begin{array}{c}
\text { true } \text { if } \Pi(\boldsymbol{p}) \mapsto\left(u^{\prime}, v^{\prime}\right) \\
\left|u^{\prime}-u\right| \leq 0.5 \wedge\left|v^{\prime}-v\right| \leq 0.5 \\
\text { false otherwise }
\end{array}\right.
$$

Let $\mathcal{N}=\left\{\boldsymbol{p}_{j} \mid \gamma\left(u, v, \boldsymbol{p}_{j}\right)\right\}$ be the set of all points that are projected on pixel $(u, v)$. As a simple heuristic to mitigate issues from occlusions, we limit the size of $\mathcal{N}$ to a maximum of 8 . If more than 8 points map onto $(u, v)$, we select only the 8 points that are closest to the camera. Then, the intensity $V(u, v)$ of the virtual image is given as the average of $N_{c}$ laser scanner intensity values $C\left(\boldsymbol{p}_{j}\right)$,

$$
V(u, v)=\frac{1}{N_{c}} \sum_{p_{j} \in \mathcal{N}} C\left(\boldsymbol{p}_{j}\right)
$$

Instead of the naive approach, more sophisticated methods can be used to obtain intensity values, for example ray casting. However, this is beyond the scope of this work.

Next, the calibration patterns are detected in the virtual image and matched to the keypoints from the camera image. These correspondences can be used to compute a second rigid body transformation $\mathcal{R}_{r}^{*}$, which we call refinement transformation. $\mathcal{R}_{r}^{*}$ is obtained by minimizing the reprojection error between corresponding keypoints as given in Eq. (6). The cost function measures the
2-D distance between a keypoint $\boldsymbol{x}_{i}$ and its corresponding transformed and projected keypoint $\hat{\boldsymbol{x}}_{i}$ in the other image.

$$
\begin{aligned}
\mathcal{R}_{r}^{*}= & \underset{\mathcal{R}_{r}}{\operatorname{argmin}} \sum_{i}\left\|\boldsymbol{x}_{i}-\Pi \mathcal{R}_{r} \hat{\Pi}^{-1}\left(\hat{\boldsymbol{x}}_{i}\right)\right\| \\
& +\left\|\hat{\boldsymbol{x}}_{i}-\hat{\Pi} \mathcal{R}_{r}^{-1} \Pi^{-1}\left(\boldsymbol{x}_{i}\right)\right\|,
\end{aligned}
$$

where $\hat{\Pi}$ denotes the projection from the point cloud to the virtual image. The inverse projections $\Pi^{-1}$ and $\hat{\Pi}^{-1}$ are obtained by solving the perspective-n-point problem. Note that there are also direct solutions to calculate rotations and translations for 3-D point correspondences, for example the method by Horn [27]. By choosing the nonlinear optimization approach, we can jointly optimize both for $\mathcal{R}_{r}^{*}$ and the transformation which relates the calibration pattern coordinate system and the camera coordinate system, thereby achieving a more accurate refinement transformation. By concatenating $\mathcal{R}_{i}^{*}$ and $\mathcal{R}_{r}^{*}$, the final spatial transformation $\mathcal{R}_{f}$ is obtained. Knowing the final rigid body transformation, it is possible to directly transform any point cloud into the coordinate system of the camera. This transformed point cloud allows the calculation of virtual amplitude images or virtual distance images that are accurately aligned with the camera image.

\section{Evaluation}

A particular benefit of a laser scanner-to-camera calibration is the ability to create ground truth for evaluating range sensors. To this end, we use two classes of range sensors as cameras: time-of-flight (ToF) sensors (Microsoft Kinect V2 and PMD CamBoard Pico Flexx) and structured light sensors (Intel RealSense R200 and the Orbbec Astra). The laser scanner is a Leica ScanStation P20 scanner.

In case of the ToF cameras, the amplitude channel is used to capture the calibration scene. For calibrating the structured light cameras to the laser scanner, we use the infrared channels with the pattern emitter either being covered or disabled. Of the RealSense R200's two infrared channels, we choose the left one as it is aligned with the distance map.

Whenever possible, we used the factory calibration of the range cameras as provided by the individual camera SDKs. For all cameras, except the Astra, these parameters could be obtained. The latter was calibrated with 60 checkerboard images using the method by Zhang [28].

In this evaluation we use the approach proposed by Geiger et al. [14] as baseline. It consists of an initialization and a refinement stage, similarly as the proposed method. Initialization is performed in a similar way as the proposed method and results in a set of rotations and translations, one of which approximately aligns the two coordinate systems. Different from our method, Geiger et al. propose to use the iterative closest point algorithm (ICP) for refining 
the initial alignment. In order to create common basis for comparison, we replace the proposed stereo calibrationbased refinement with the ICP-based refinement. To this end, we generate 3-D coordinates from the camera intrinsics and the checkerboard coordinates as suggested by the authors.

The setup of our experiments and the used data is described in Section 4.1. In Section 4.2, we present qualitative results for the proposed method. In Section 4.3, we present quantitative results on the evaluation scenes. Section 4.4 provides additional insights on the impact of the proposed refinement step. An evaluation of the measurement accuracy of a ToF (Kinect V2) and a structured light camera (RealSense R200) conclude the evaluation in Section 4.5.

\subsection{Experimental setup and data}

We captured five different scenes with the same spatial relation: a calibration scene that shows the four patterns, an evaluation scene with rearranged patterns, and three general scenes with different objects. The individual point clouds contain $\approx 6.2$ million points, sampled from a volume of approximately $230 \times 150 \times 95 \mathrm{~cm}$.

In the calibration and evaluation scene, four checkerboard patterns with different dimensions and a different number of corners are captured:

- $6 \times 5$ inner corners, $66.67 \mathrm{~mm}$ corner spacing

- $6 \times 7$ inner corners, $50 \mathrm{~mm}$ corner spacing

- $6 \times 9$ inner corners, $44 \mathrm{~mm}$ corner spacing

- $8 \times 7$ inner corners, $50 \mathrm{~mm}$ corner spacing

All patterns are printed on rigid boards to avoid errors from bending calibration patterns. Captured images are averaged for 100 frames to reduce the impact of measurement noise. The spatial relation between the laser scanner and the cameras is calculated on the first scene.

In all amplitude, intensity, and virtual images shown in the evaluation, the pixel values are normalized to values between 0 and 1 for better comparison.

\subsection{Qualitative evaluation results}

We illustrate the performance of the proposed method by comparing virtual reflectance images with observed images. The calibrations between the individual cameras and the point cloud are calculated on the first scene. Then, calibration data is used to generate virtual images of the evaluation scene for each camera. For comparison, each camera has been calibrated with the proposed method and the baseline method to the point cloud.

Figure 2 shows the difference between the observed camera images and the virtual images for the baseline method (denoted as "ICP") and for the proposed method (denoted as "Proposed"). Wrong spatial relations show as additional edges in the scene, whereas all edges in the scene coincide for an accurate transformation. It is important to emphasize that the magnitude of the pixel differences is not caused by misalignments, but by the internal conversion of the incoming light to intensities of the different sensors.

When calculating the spatial parameters with the baseline method, small offsets between corresponding edges can be observed (see Fig. 2a, e, and g). In contrast, for the proposed method, the virtual and observed images accurately coincide for all cameras. A visual comparison of the calibration results of the CamBoard Pico Flexx is difficult due to the low sensor resolution and the low reflectance of the black checkerboard patches. In these areas, the CamBoard Pico Flexx does not provide amplitude values, as only a small portion of the emitted light is reflected back to the sensor.

\subsection{Quantitative results}

The reprojection error is a common choice to evaluate the quality of a stereo calibration result. Thus, we compare the checkerboard positions in the camera image and in the virtual image. In this experiment, the system is calibrated on the first scene. Hereafter, the reprojection errors are calculated on the evaluation scene. As the virtual image is generated from the perspective of the camera, we can directly compare the 2-D positions of the keypoints which are returned by the checkerboard detection algorithm.

For assessing the impact of the misalignments in 3-D, we reproject the keypoints based on the intrinsic parameters and known pattern dimensions. Similarly, as in the 2-D case, we can directly compute the differences between corresponding 3-D world coordinates.

The results of this comparison are shown in Table 1. With the proposed method, we observe mean errors in corresponding 3 -D coordinates between $\approx 1$ to $3 \mathrm{~mm}$, depending on the used camera. When relying only on 3-D information, like in the ICP variant, the measured errors are at least three times as large as for the proposed approach.

The calibration errors of the proposed method are within the expectation of typical stereo calibrations. The authors of the pattern detector report 3-D measurement errors between 1 and $7 \mathrm{~mm}$, depending on the sensor resolution [26].

\subsection{Influence of the refinement transformation}

In this section we demonstrate the importance of the refinement via stereo calibration. During initialization, the centroids of the planar segments that represent the checkerboard patterns in the laser scanner's point cloud are used to calculate the initial transformations. If these centroids are not perfectly accurate, then the resulting 


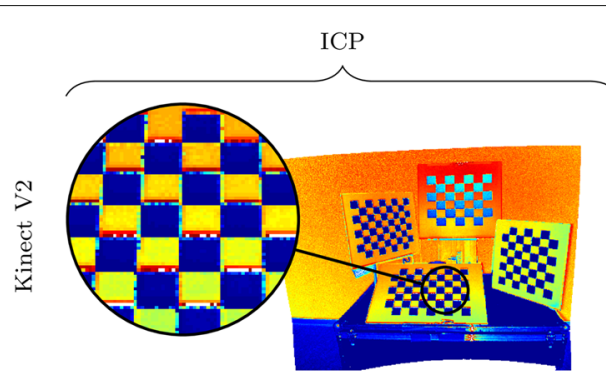

a

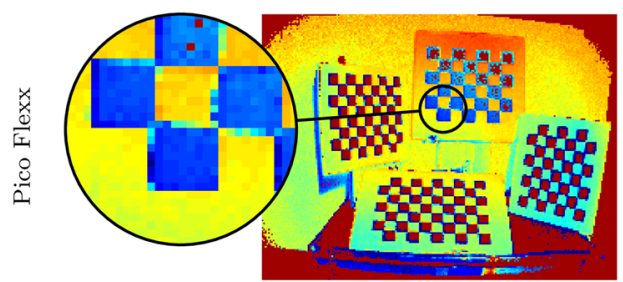

C

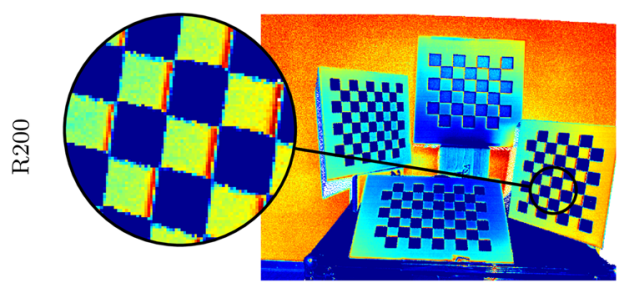

e

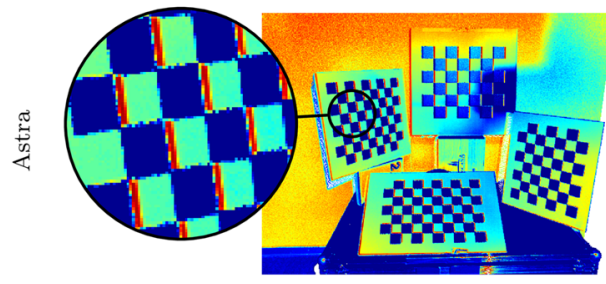

g

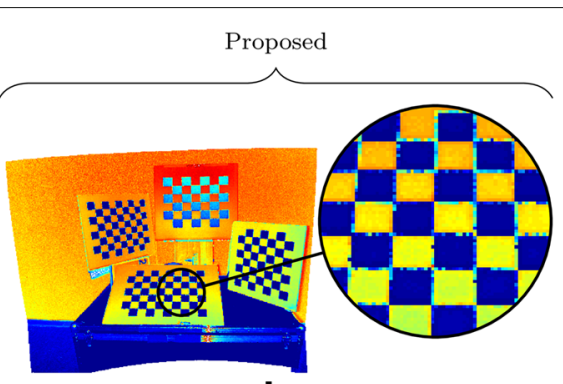

b

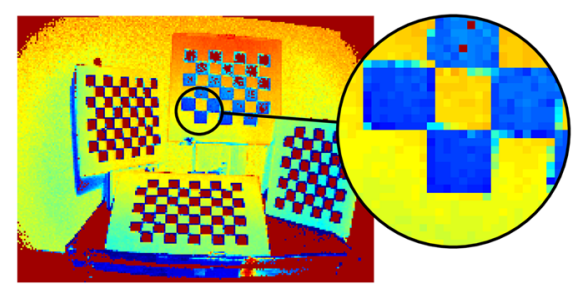

d

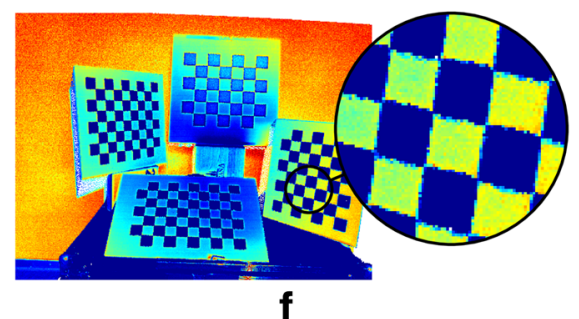

f

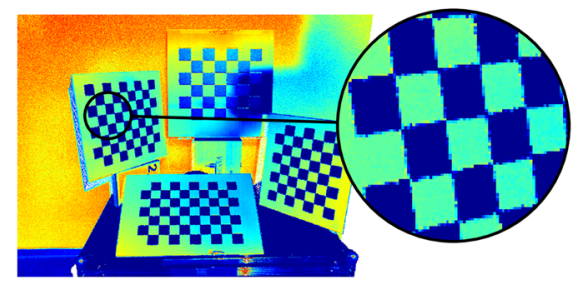

h

Fig. 2 Difference of observed image and the corresponding virtual images for the two evaluated approaches. The difference images (a), (c), (e) and $(\mathbf{g})$ have been calculated with virtual images generated with calibrations from the ICP-based approach. The figures $(\mathbf{b}),(\mathbf{d})$, (f) and (h) show overlays which have been calculated with calibrations from the proposed method. For the baseline method, misalignments can be observed in examples

(a), (e) and (f). In contrast, no misalignments identified if the proposed method is used for calibration

initial transformation has only limited accuracy. The centroid is given by the mean coordinate of all points that belong to the segment, and thus sensitive to segmentation errors. If the segmentation for example contains points of the supporting surface of the calibration pattern, or if the segmentation is not completely homogeneous, then the centroid will be off-center.

In Fig. 3a, double edges at the checkerboard quads indicate that the two images are not accurately aligned. Figure $3 \mathrm{~b}$ shows the difference image of the Kinect's

Table 1 Mean calibration error and standard deviation for four cameras in 2-D and 3-D for two calibration approaches

\begin{tabular}{lllll}
\hline Camera & ICP 2-D (px) & Proposed 2-D (px) & ICP 3-D (cm) & Proposed 3-D (cm) \\
\hline Kinect V2 & $1.172 \pm 0.492$ & $0.176 \pm 0.084$ & $1.113 \pm 0.108$ & $0.267 \pm 0.064$ \\
CamBoard Pico Flexx & $2.309 \pm 0.509$ & $0.305 \pm 0.125$ & $0.930 \pm 0.124$ & $0.319 \pm 0.134$ \\
Astra & $0.491 \pm 0.232$ & $0.418 \pm 0.119$ & $1.135 \pm 0.444$ & $0.126 \pm 0.038$ \\
RealSense R200 & $1.124 \pm 0.653$ & $0.252 \pm 0.121$ & $0.880 \pm 0.285$ & $0.160 \pm 0.109$ \\
\hline
\end{tabular}




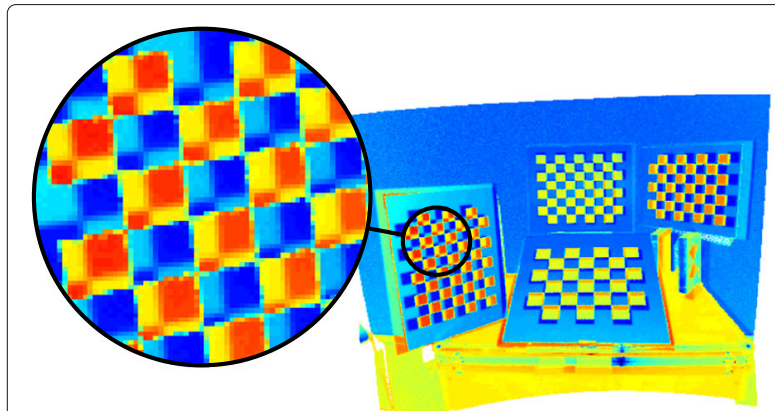

a Difference between intial virtual and observed images

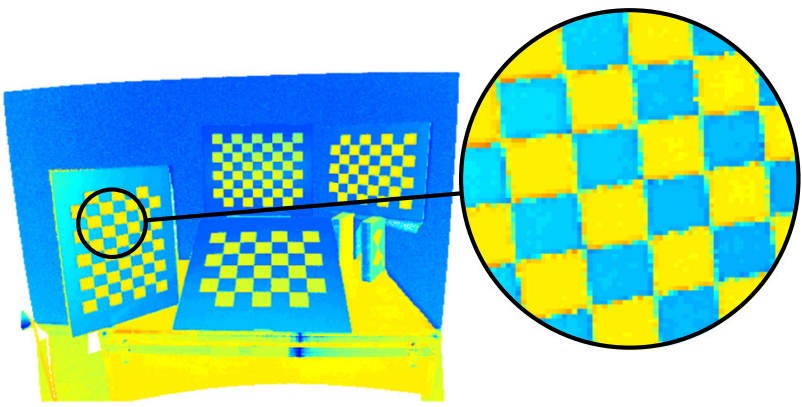

b Difference between refined virtual and observed images

Fig. 3 Impact of refinement for the calibration scene and the Kinect V2. a Difference between the virtual reflectance image and the observed image. Inaccuracies in the initialization cause edges to appear twice. b Difference image between the final reflectance image and the amplitude image of the camera: all edges accurately coincide

amplitude image and the virtual reflectance image after refinement. In the refined result, no double edges can be observed. Instead, all checkerboard quads as well as the borders of the pattern boards are accurately aligned.

\subsection{Range camera evaluation}

For this experiment, we use the calibration results to generate ground truth distance data for the three general scenes. Depending on the focus of the study, the scenes have to be designed differently. The primary interest of the following experiment is to evaluate the absolute measurement accuracy of the range cameras with a certain volume of interest. Furthermore, we set up the scene such that several characteristics of the different range camera technologies can be illustrated. In this evaluation, we present as an example evaluation results for the Kinect V2 and the RealSense R200 camera, i.e, representatives of both classes of range cameras.

The setup consists of several objects which are positioned in front of the sensors: boxes, cylinders, etc. Each scene is captured first with the laser scanner then with the two cameras, to exclude mutual interference. Then, the individual range camera measurements are compared to the reference distance images which have been generated from laser scanner data. For visualizing the measurement errors, we subtract the observed distance image from the virtual distance image.

In Fig. 4 we demonstrate some of the characteristic properties of each sensor type on one of the general scenes. To investigate the dependency of measurement error and distance, we combine the result of the three general scenes and plot the errors for a region of interest as shown in Fig. 5. The mean accuracy is calculated for $1-\mathrm{cm}$ bins and plotted in black. Gray dots represent individual measurements.

\subsubsection{Kinect V2}

The ToF sensor provides dense distance measurements for all pixels which are properly illuminated (see Fig. 4b). In Fig. 4c, the measurement errors for all pixels which lie in the common field of view of the laser scanner and the range camera are illustrated. Two characteristic errors can be observed in this figure: an amplitude-dependent error and multi-path effects [7]. The amplitude-dependent error can be observed best in the lower right corner of the image in the area of the calibration target. Even though the surface of this target is flat, a clear change of distances can be observed between high- and lowreflectivity regions. Multi-path can be observed best in the central image region. In this area, the emitted light can easily hit multiple regions one after another before being reflected back to the camera. Especially for pixels that belong to the flat surface of the box, the acute viewing angle fosters a comparably large impact of multi-path effects.

The results shown in Fig. 5 support this insight. Most of the objects in the scene can be found within the range from 1.5 to $1.85 \mathrm{~m}$. The objects in the first row of the scene can be expected to suffer only marginally from multi-path effects as there are only very few small surfaces which could reflect the light such that it will return to the camera. In contrast, the central region of the scene contains more objects, and thus more surfaces which can create non-direct paths back to the camera. This can also be observed in Fig. 4g, where the absolute mean error changes drastically for distances that contain the center of the scene (see highlighted areas in Fig. $5 \mathrm{a}-\mathrm{c}$ ). For larger distances, which contain the well-reflecting background, the measurement accuracy increases again. For all areas which are not affected by multi-path, and which are well illuminated, the measurement error matches earlier reported values [7]. 


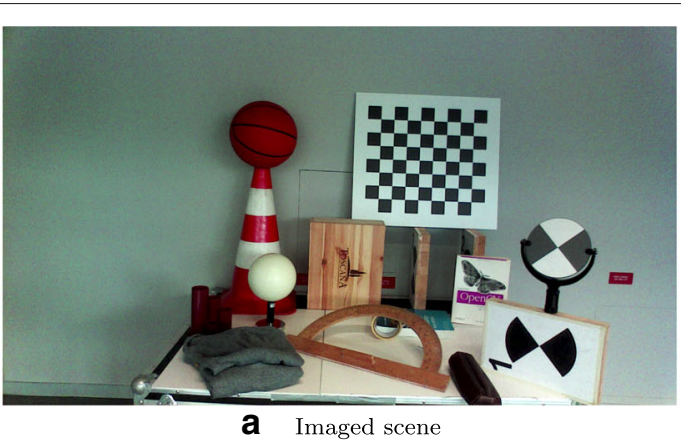

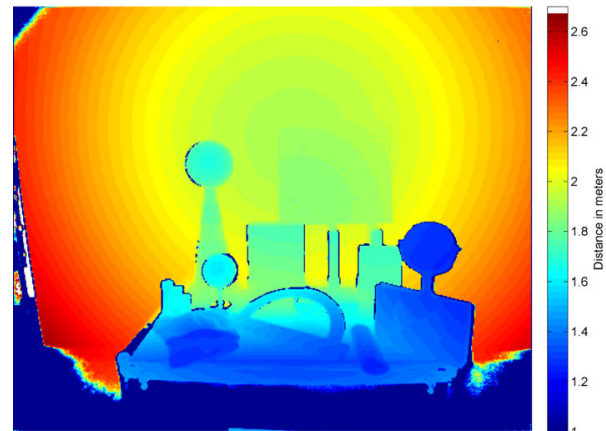

b Kinect V2 distance image

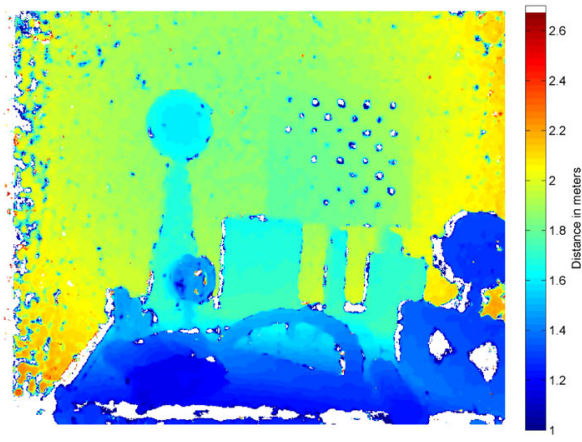

d RealSense R200 distance image

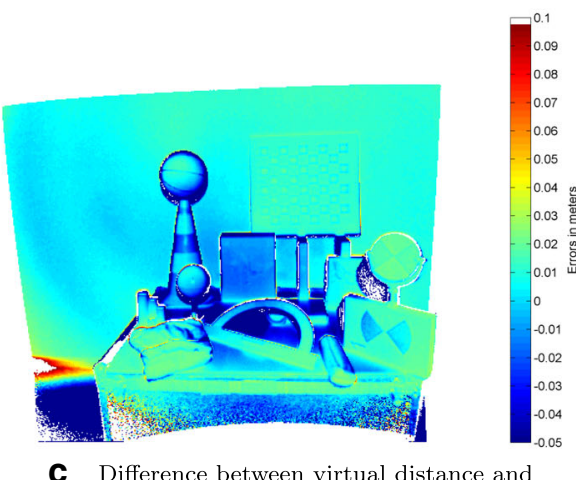

Kinect V2 distance image

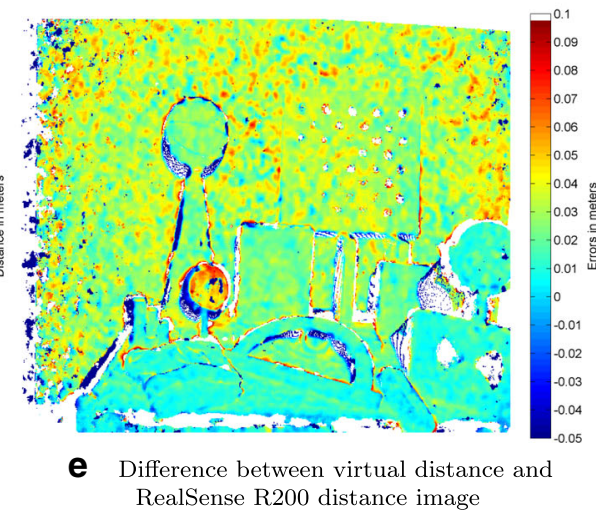

Fig. 4 Per-pixel accuracy evaluation for one of the general scenes. Distances and measurement errors are given in meters and encoded as colors. a Imaged scene. b Kinect V2 distance image c depth error for Kinect V2. d RealSense R200 distance image. e depth error for RealSense R200

\subsubsection{RealSense $\mathbf{R} 200$}

In this section, characteristic errors of the RealSense R200 structured light camera are investigated. Stereo block matching and the subsequent internal processing causes the speckle-like pattern that can be observed best in the planar background region of Fig. 4e. Block matching also causes fringes at sharp borders, e.g., at the borders of the spheres. Similarly as the time-of-flight camera, this sensor also relies on the requirement that the emitted light is reflected back to the cameras. If the imaged surface does not reflect the light in the spectrum of the emitter, then no or only inaccurate measurements are possible. These effects can be observed in the lower left sphere (inaccurate measurements) and at the lower right calibration target. A characteristic of structured-light cameras is that the baseline between projector and observing camera introduces occluded image regions, seen best at the disc on the right side of the image.

Another error characteristic of stereo structured light cameras is the fact that the theoretical depth resolution decreases with the distance between camera and measured point [29]. This observation can also be made in Fig. 4h. While the accuracy for near regions is approximately $1 \mathrm{~cm}$, it decreases steadily as the distance increases. For background pixels, the average accuracy drops to $\approx 3.5 \mathrm{~cm}$ (highlighted in blue). 


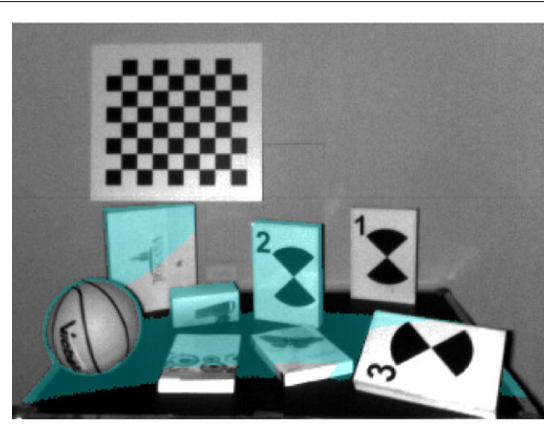

a $\quad$ Kinect V2 scene 1

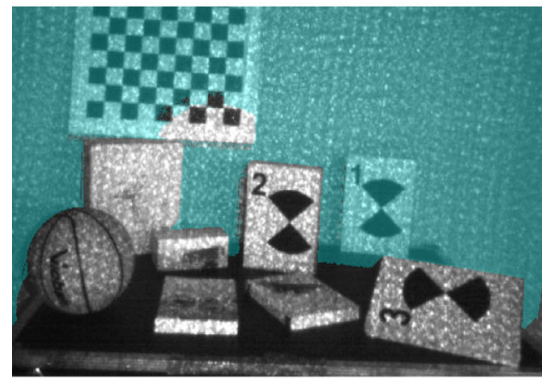

d RealSense R200 scene 1

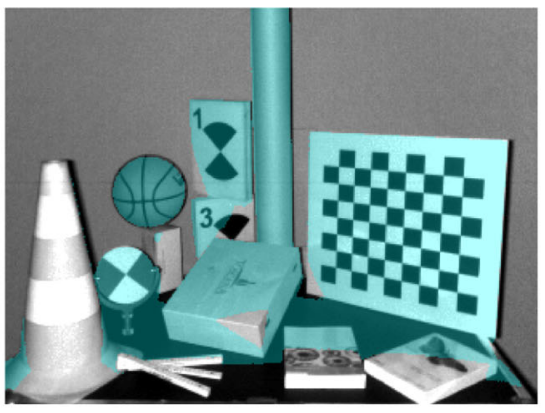

b Kinect V2 scene 2

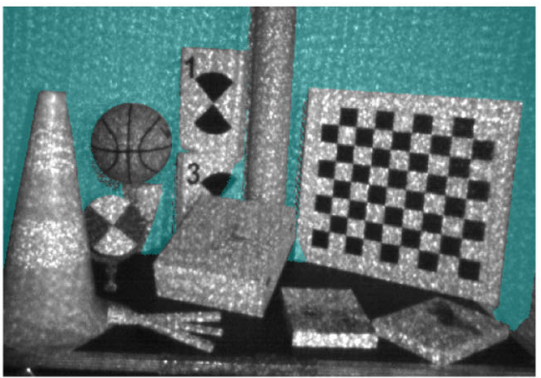

e RealSense R200 scene 2

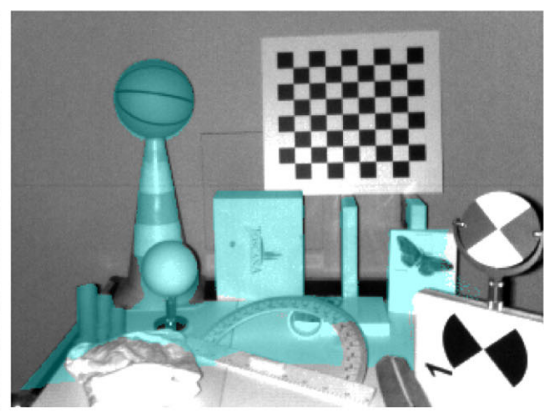

C Kinect V2 scene 3

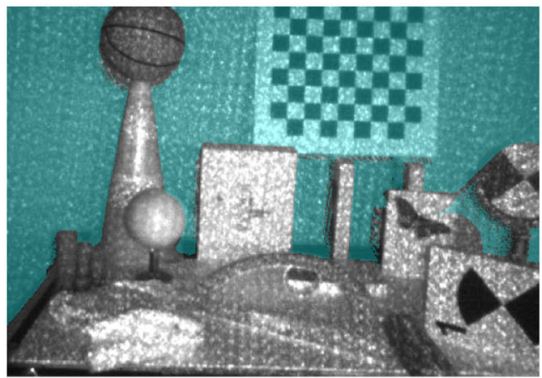

f RealSense R200 scene 3

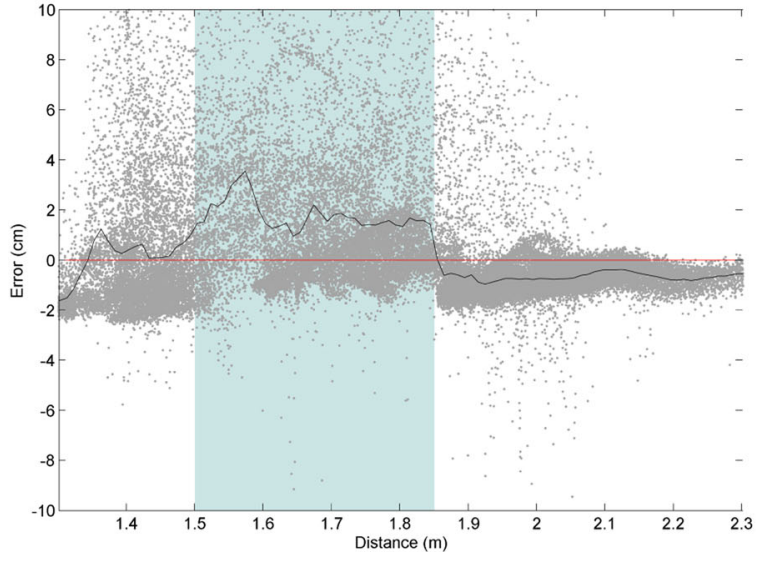

g Kinect V2

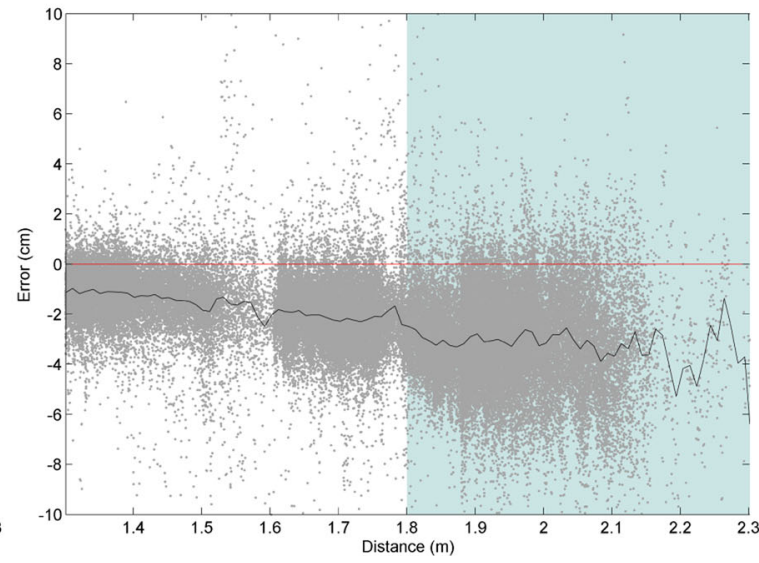

h RealSense R200

Fig. 5 Measurement error with respect to distance. a-c Evaluated ROI of the three scenes from the perspective of the Kinect V2 camera. d-f Respective ROls for the RealSense R200 sensor. $\mathbf{g}$, h Mean measurement error with respect to distance. Gray dots depict individual measurements. The black line represents the mean measurement error within $1 \mathrm{~cm}$. The red line marks the zero-level to help reading the plots. The light blue overlays highlight interesting distance ranges which are discussed in the evaluation. (Kinect V2: 1.5 to 1.85 m, RealSense R200: 1.8 to $2.3 \mathrm{~m}$ )

\section{Conclusion}

We presented a novel method for finding the spatial relation between a camera and laser scanner based on stereo calibration. The algorithm enables the user to calibrate the laser scanner to a camera with high accuracy using only a single shot of a calibration scene.

In our evaluation, we compare the proposed method to a similar, calibration pattern-based approach and show that our method achieves notably more accurate calibration results. In terms of the reprojection error, our method outperforms the baseline at least by a factor of three. Furthermore, we show that the calibration results compare very well to standard stereo calibration algorithms, although the sensory data to our method is very heterogeneous. For corresponding keypoints of the observed and virtual images, the misalignments account for less than 0.2 pixels. In world coordinates, this results in errors of less than $1.3 \mathrm{~mm}$. 
The applicability of the method is demonstrated in the context of range camera evaluation. Here, we use the method to investigate the measurement errors of a timeof-flight and a structured light camera: the Microsoft Kinect V2 and the Intel RealSense R200. In this evaluation, we can showcase several error sources which are characteristic to the different range sensing technologies.

\section{Acknowledgements}

We thank PMD technologies for providing the Camboard Pico Flexx time-of-flight camera which has been used in this evaluation.

\section{Authors' contributions}

PF performed the primary development of the algorithm, designed the evaluation, and wrote the intial draft of the manuscript. CP helped with the data acquisition and provided the laser scanner. AM and CR supervised the work. CR also played an essential role in drafting and refining the final manuscript. All authors read and approved the manuscript.

\section{Competing interests}

The authors declare that they have no competing interests.

\section{Publisher's Note}

Springer Nature remains neutral with regard to jurisdictional claims in published maps and institutional affiliations.

\section{Author details}

${ }^{1}$ Pattern Recognition Lab, University of Erlangen - Nuremberg, Martensstrasse 3, Erlangen, Germany. ${ }^{2}$ Metrilus GmbH, Henkestrasse 91, Erlangen, Germany. ${ }^{3}$ Ostbayerische Technische Hochschule Regensburg, Pruefeninger Strasse 58, Regensburg, Germany.

Received: 31 March 2017 Accepted: 18 October 2017

Published online: 10 November 2017

\section{References}

1. Buck S, Hanten R, Bohlmann K, Zell A (2016) Generic 3D obstacle detection for AGVs using time-of-flight cameras. In: 2016 IEEE/RSJ International Conference on Intelligent Robots and Systems (IROS). pp 4119-4124

2. Smith T (2016) Astrobee: A New Platform for Free-Flying Robotics on the International Space Station. In: Proceedings of the International Symposium on Artificial Intelligence, Robotics and Automation in Space (i-SAIRAS 2016)

3. Klionovska K, Benninghoff H (2017) Initial Pose Estimation using PMD Sensor during the Rendezvous Phase in On-Orbit Servicing Missions. In: 27th AAS/AIAA Space Flight Mechanics Meeting

4. Leo M, Natale A, Del-Coco M, Carcagnì P, Distante C (2017) Robust Estimation of Object Dimensions and External Defect Detection with a Low-Cost Sensor. J Nondestruct Eval 36(1)

5. Rauscher G, Dube D, Zell A (2014) A Comparison of 3D Sensors for Wheeled Mobile Robots. In: 2014 International Conference on Intelligent Autonomous Systems (IAS-13). Padova

6. Yang L, Zhang L, Dong H, Alelaiwi A, Saddik AE (2015) Evaluating and Improving the Depth Accuracy of Kinect for Windows v2. EEE Sensors J 15(8):4275-4285

7. Fuersattel P, Placht S, Balda M, Schaller C, Hofmann H, Maier A, et al. (2016) A Comparative Error Analysis of Current Time-of-Flight Sensors. IEEE Trans Comput Imaging 2(1):27-41

8. Wasenmüller O, Stricker D (2017) Comparison of Kinect v1 and v2 Depth Images in Terms of Accuracy and Precision. In: Asian Conference on Computer Vision Workshop Asian Conference on Computer Vision Workshop (ACCV)

9. Nair R, Meister S, Lambers M, Balda M, Hofmann H, Kolb A, et al. (2013) Ground Truth for Evaluating Time of Flight Imaging. In: Time-of-Flight and depth imaging: Sensors, algorithms, and applications : Dagstuhl 2012 Seminar on Time-of-Flight Imaging and GCPR 2013 Workshop on Imaging New Modalities. pp 52-74

10. Zhang Q, Pless R (2004) Extrinsic calibration of a camera and laser range finder (improves camera calibration). In: 2004 IEEE/RSJ International Conference on Intelligent Robots and Systems (IROS). pp 2301-2306
11. Kassir A, Peynot T (2010) Reliable automatic camera-laser calibration. In: Proceedings of the 2010 Australasian Conference on Robotics \& Automation

12. Zhou L (2014) A New Minimal Solution for the Extrinsic Calibration of a 2D LIDAR and a Camera Using Three Plane-Line Correspondences. IEEE Sensors J 14(2):442-454

13. Unnikrishnan R, Hebert M (2005) Fast Extrinsic Calibration of a Laser Rangefinder to a Camera. Pittsburgh

14. Geiger A, Moosmann F, Car O, Schuster B (2012) Automatic camera and range sensor calibration using a single shot. In: 2012 IEEE International Conference on Robotics and Automation (ICRA). pp 3936-3943

15. Ha JE (2012) Extrinsic calibration of a camera and laser range finder using a new calibration structure of a plane with a triangular hole. Int J Control Autom Syst 10(6):1240-1244

16. Hoang VD, Cá D, Jo KH (2014) Simple and Efficient Method for Calibration of a Camera and 2D Laser Rangefinder. In: Hutchison D, Kanade T, Kittler J, Kleinberg JM, Mattern F, Mitchell JC, et al (eds). Intelligent Information and Database Systems, vol 8397 of Lecture Notes in Computer Science. Springer International Publishing. pp 561-570

17. Gong X, Lin Y, Liu J (2013) 3D LIDAR-camera extrinsic calibration using an arbitrary trihedron. Sensors 13(2):1902-1918

18. Moghadam P, Bosse M, Zlot R (2013) Line-based extrinsic calibration of range and image sensors. In: 2013 IEEE International Conference on Robotics and Automation (ICRA). pp 3685-3691

19. Taylor Z, Nieto J (2012) A Mutual Information Approach to Automatic Calibration of Camera and Lidar in Natural Environments. In: Proceedings of Australasian Conference on Robotics and Automation (ACRA). pp 31-39

20. Taylor Z, Nieto J (2013) Automatic calibration of lidar and camera images using normalized mutual information. University of Sydney, Australia

21. Pandey G, McBride JR, Savarese S, Eustice RM (2015) Automatic Extrinsic Calibration of Vision and Lidar by Maximizing Mutual Information. J Field Robot 32(5):696-722

22. Levinson J, Thrun S (2013) Automatic Online Calibration of Cameras and Lasers. In: Proceedings of Robotics: Science and Systems. Berlin

23. Scott T, Morye AA, Pinies P, Paz LM, Posner I, Newman P (2015) Exploiting known unknowns: Scene induced cross-calibration of lidar-stereo systems. In: 2015 IEEE/RSJ International Conference on Intelligent Robots and Systems (IROS). pp 3647-3653

24. Poppinga J, Vaskevicius N, Birk A, Pathak K (2008) Fast plane detection and polygonalization in noisy 3D range images. In: 2008 IEEE/RSJ International Conference on Intelligent Robots and Systems. pp 3378-3383

25. Holz D, Behnke S (2014) Approximate triangulation and region growing for efficient segmentation and smoothing of range images. Robot Auton Syst 62(9):1282-1293

26. Placht S, Fürsattel P, Mengue EA, Hofmann H, Schaller C, Balda M, et al. (2014) ROCHADE: Robust Checkerboard Advanced Detection for Camera Calibration. In: Fleet D, Pajdla T, Schiele B, Tuytelaars T (eds). Computer Vision - ECCV 2014. vol. 8692 of Lecture Notes in Computer Science. Springer International Publishing, Cham. pp 766-779

27. Horn BKP (1987) Closed-form solution of absolute orientation using unit quaternions. J Opt Soc Am A 4(4):629-642

28. Zhang Z (2000) A flexible new technique for camera calibration. IEEE Trans Pattern Anal Mach Intell 22(11):1330-1334

29. Kytö M, Nuutinen M, Oittinen P (2011) Method for measuring stereo camera depth accuracy based on stereoscopic vision. In: Beraldin JA, Cheok GS, McCarthy MB, Neuschaefer-Rube U, Baskurt AM, McDowall IE, et al. (eds). Three-Dimensional Imaging, Interaction, and Measurement. SPIE Proceedings. SPIE. p 78640I 